\title{
Universality in three-frequency resonances
}

\author{
Julyan H. E. Cartwright, ${ }^{1, *}$ Diego L. González, ${ }^{2, \dagger}$ and Oreste Piro ${ }^{3,+}$ \\ ${ }^{1}$ Instituto Andaluz de Ciencias de la Tierra, IACT (CSIC-UGR), E-18071 Granada, Spain \\ ${ }^{2}$ Istituto Lamel, CNR, I-40129 Bologna, Italy \\ ${ }^{3}$ Institut Mediterrani d'Estudis Avançats, IMEDEA (CSIC-UIB), E-07071 Palma de Mallorca, Spain
}

(Received 7 August 1998)

\begin{abstract}
We investigate the hierarchical structure of three-frequency resonances in nonlinear dynamical systems with three interacting frequencies. We hypothesize an ordering of these resonances based on a generalization of the Farey tree organization from two frequencies to three. In experiments and numerical simulations we demonstrate that our hypothesis describes the hierarchies of three-frequency resonances in representative dynamical systems. We conjecture that this organization may be universal across a large class of three-frequency systems.
\end{abstract} [S1063-651X(99)14803-7]

PACS number(s): 05.45.-a

Nonlinear systems with two competing frequencies show resonances or lockings, in which the system locks into a resonant periodic response which has a rational frequency ratio [1]. The locking increases with nonlinearity, from none in the linear regime, to a critical situation where the system is everywhere resonant. The subcritical system has quasiperiodic responses between different lockings, while at supercritical values of the nonlinearity, chaotic as well as periodic and quasiperiodic responses may occur. Resonances have been investigated theoretically and experimentally in many nonlinear systems, and their distribution in parameter space in the form of a devil's staircase is now well understood, from the number theoretical concept of Farey trees [2-7]. However, all this applies to resonances generated by the interaction of two frequencies. Far less is known, by comparison, when there are three or more interacting frequencies.

Adding another frequency allows new phenomena to take place. Now as well as (two-frequency) resonance as before, there is a further possibility: three-frequency resonance, also known as weak resonance or partial mode locking. Threefrequency resonances are given by the nontrivial solutions of the equation $a f_{0}+b f_{1}+c f_{2}=0$, where $a, b$, and $c$ are integers, $f_{1}$ and $f_{2}$ are the forcing frequencies, and $f_{0}$ is the resonant response. They form a web in the parameter space of the frequencies [8-11]. In this paper, we hypothesize a local ordering of three-frequency resonances based on generalizing the Farey tree of two-frequency systems to three frequencies. We perform experiments and numerical simulations to show that our hypothesis is justified in representative dynamical systems with three interacting frequencies: a quasiperiodically forced circle map, a pair of parametrically coupled forced nonlinear oscillators, and an experimental system consisting of an electronic circuit of forced phaselocked loops. Our observations lead us to conjecture that the

*URL: WWW http://formentor.uib.es/ julyan. Electronic address: julyan@hp1.uib.es

${ }^{\dagger}$ Electronic address: diego@indra.lamel.bo.cnr.it

${ }^{\ddagger}$ URL: WWW http://www.imedea.uib.es/ piro. Electronic address: piro@imedea.uib.es ordering we predict may be universal in a large class of dynamical systems with three interacting frequencies.

Firstly, we revise continued fractions and the Farey tree for the case of two frequencies. Consider a two-frequency system with autonomous frequency $f_{0}$ and external frequency $f_{1}$. Let $\widetilde{f}=f_{1} / f_{0}$. The aim is to define a sequence of rationals that converges to $\widetilde{f}$. Strong convergence [12] is defined for rational fractions $p_{i} / q_{i},\left(p_{i}, q_{i}\right) \in \mathbb{Z}$ as

$$
\left\|\tilde{f}-\frac{p_{i}}{q_{i}}\right\|=\left|q_{i} \tilde{f}-p_{i}\right| .
$$

$p_{n} / q_{n}$ is a best rational approximation if

$$
\left\|\tilde{f}-\frac{p_{n}}{q_{n}}\right\|<\left\|\tilde{f}-\frac{p_{i}}{q_{i}}\right\|
$$

for all $\left(p_{i}, q_{i}\right)$ for any $q_{i} \leqslant q_{n}$. Given $\widetilde{f}, p_{n}$ and $q_{n}$ are obtained by expanding $\tilde{f}$ in continued fractions $\tilde{f}$ $=\left(a_{1}, a_{2}, a_{3}, \ldots\right)$, and truncating the expansion as $p_{n} / q_{n}$ $=\left(a_{1}, a_{2}, a_{3}, \ldots a_{n}\right)$ [13]. The $p_{n} / q_{n}$ are then the strong convergents of $\tilde{f}$. They give the sequence of fractions with lowest monotonically increasing denominators that converges to $\widetilde{f}$.

The physically motivated hypothesis invoked to explain the local ordering of the hierarchy of (two-frequency) resonances is that the larger the denominator, the smaller the plateau. The fraction with smallest denominator between $p / q$ and $r / s$, if they are sufficiently close that $|q r-p s|=1$, when they are called adjacents, is $(p+r) /(q+s)$. This fraction, known as the mediant, is then the most important resonance in the interval. Repeatedly performing the mediant operation

$$
\frac{p}{q} \oplus \frac{r}{s}=\frac{p+r}{q+s}
$$

on a pair of adjacent rational numbers, we obtain a Farey tree. The Farey tree provides a qualitative local ordering of two-frequency resonances, and gives rise to a structure of plateaus at all rationals known as the devil's staircase. The 
(a)
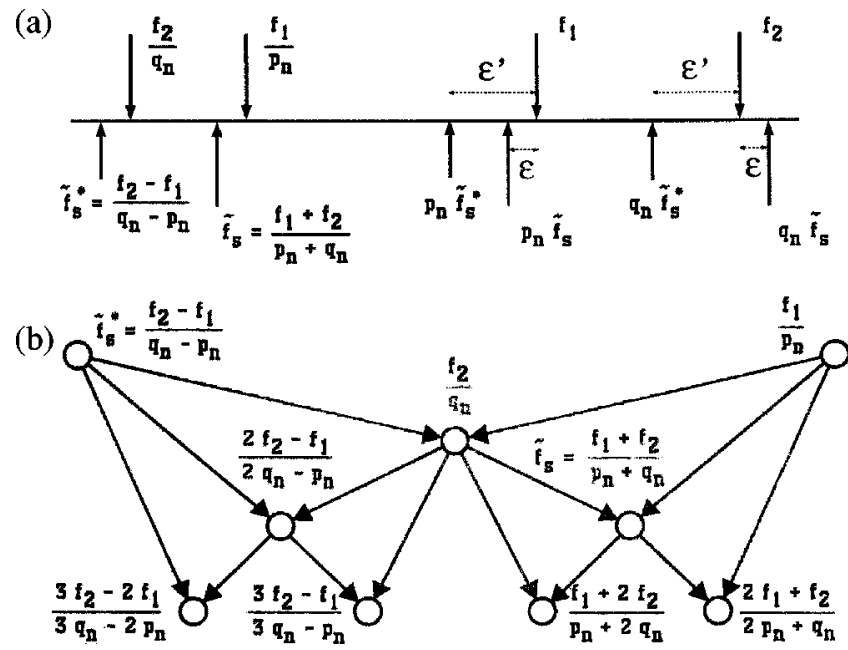

FIG. 1. (a) Schematic diagram of the frequency line, showing the relative position of $\widetilde{f}_{s}$ and $\tilde{f}_{s}^{*}$ and their respective $p_{n}, q_{n}$ harmonics that approximate $f_{1}$ and $f_{2}$ at equal distance. (b) Generalized Farey tree starting from the adjacents $f_{1} / p_{n}$ and $\tilde{f}_{s}^{*}$. The first mediant is $f_{2} / q_{n}$; at the second level we obtain $\widetilde{f}_{s}$ and $\left(2 f_{2}\right.$ $\left.-f_{1}\right) /\left(2 q_{n}-p_{n}\right)$, and so on.

devil's staircase in turn is the skeleton for the layout of the resonances in parameter space as Arnold tongues [2-7].

Now consider the case of three frequencies, one internal $f_{0}$, and two external $f_{1}$ and $f_{2}$. We may divide through by the autonomous frequency $f_{0}$, to give $f_{1}^{\dagger}=f_{1} / f_{0}$, and $f_{2}^{\dagger}$ $=f_{2} / f_{0}$. We aim to come up with two convergent sequences of rational numbers with the same denominator, $p_{n} / k_{n}$ and $q_{n} / k_{n}$, which are strong convergents to $f_{1}^{\dagger}$ and $f_{2}^{\dagger}$, respectively. As before, strong convergence is defined as

$$
\left\|\left(f_{1}^{\dagger}, f_{2}^{\dagger}\right)-\left(\frac{p_{i}}{k_{i}}, \frac{q_{i}}{k_{i}}\right)\right\|=\left|k_{i}\left(f_{1}^{\dagger}, f_{2}^{\dagger}\right)-\left(p_{i}, q_{i}\right)\right| .
$$

Thus $\left(p_{n} / k_{n}, q_{n} / k_{n}\right)$ are best rational approximants if

$$
\left\|\left(f_{1}^{\dagger}, f_{2}^{\dagger}\right)-\left(\frac{p_{n}}{k_{n}}, \frac{q_{n}}{k_{n}}\right)\right\|<\left\|\left(f_{1}^{\dagger}, f_{2}^{\dagger}\right)-\left(\frac{p_{i}}{k_{i}}, \frac{q_{i}}{k_{i}}\right)\right\|
$$

for all triplets of integers $\left(p_{i}, q_{i}, k_{i}\right)$ for any $k_{i} \leqslant k_{n}$. So we may write

$$
\begin{aligned}
& \varepsilon_{1}=\left\|\frac{p_{n}}{k_{n}}-f_{1}^{\dagger}\right\|=\left|k_{n} f_{1}^{\dagger}-p_{n}\right|, \\
& \varepsilon_{2}=\left\|\frac{q_{n}}{k_{n}}-f_{2}^{\dagger}\right\|=\left|k_{n} f_{2}^{\dagger}-q_{n}\right|,
\end{aligned}
$$

where we wish to obtain the integers $p_{n}, q_{n}$, and $k_{n}$. This general problem has not been solved [14,15]; however, we may set $\varepsilon_{1}=\varepsilon_{2}$, so that both approximations should be equally good or bad. If we do this, we can equate $\mid k_{n} f_{1}^{\dagger}$ $-p_{n}|=| k_{n} f_{2}^{\dagger}-q_{n} \mid$, and ask what is $k_{n}$. There are two solutions $k_{n}=\left(q_{n} \pm p_{n}\right) /\left(f_{2}^{\dagger} \pm f_{1}^{\dagger}\right)$. At this point we must remember that $k_{n}$ is an integer, so these solutions require that the frequencies be rescaled by $f_{2}^{\dagger} \pm f_{1}^{\dagger}$. For which we define for the first solution $\tilde{f}_{1}=f_{1}^{\dagger} /\left(f_{1}^{\dagger}+f_{2}^{\dagger}\right), \quad \tilde{f}_{2}=f_{2}^{\dagger} /\left(f_{1}^{\dagger}+f_{2}^{\dagger}\right)$, and (a)

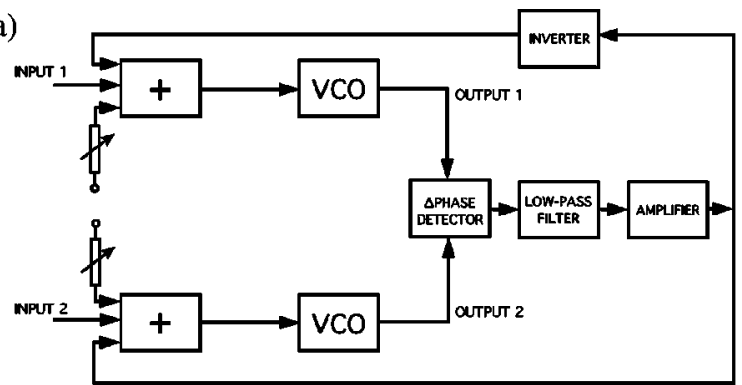

(b)

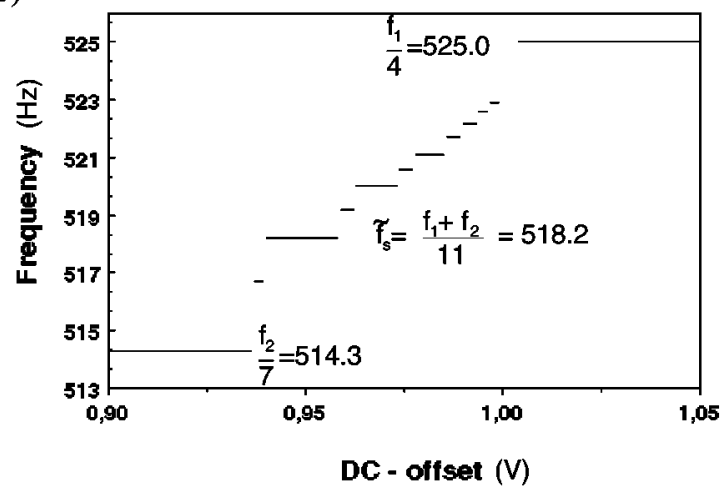

(c)

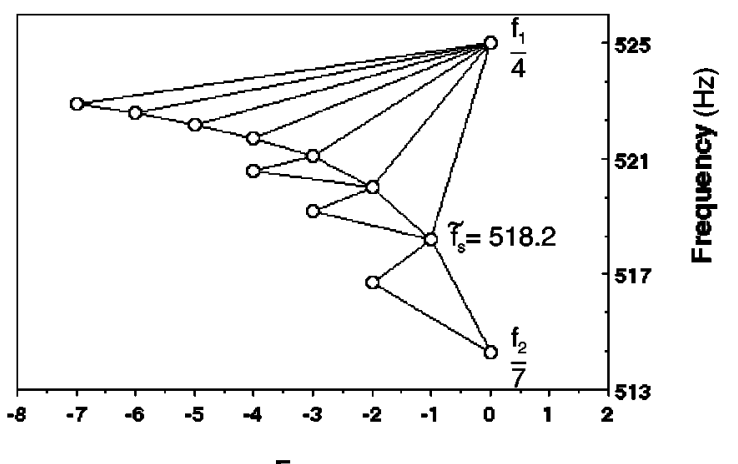

Farey sum

FIG. 2. (a) The electronic circuit in block-diagram form. Two coupled voltage-controlled oscillators (VCO's) are forced with two independent external forces. (b) Three-frequency devil's staircase for the circuit. The straight lines correspond to intervals of the $\mathrm{dc}$ offset within which the fundamental frequency of the output remains constant. We varied the dc offset of input 1 in steps of $1 \mathrm{mV}$ in the interval $0.90 \mathrm{~V}$ to $1.05 \mathrm{~V}$, which corresponds to frequency responses of the circuit between $514.28 \mathrm{~Hz}$ and $525.00 \mathrm{~Hz}$. Only intervals with a stability width greater than $2 \mathrm{mV}$ are plotted. The external frequencies are fixed at $2100 \mathrm{~Hz}$ and $3600 \mathrm{~Hz}$. (c) The generalized Farey tree predicts the organization of all the frequency values observed in (b).

similarly for the other solution $\tilde{f}_{1}^{*}=f_{1}^{\dagger} /\left(f_{2}^{\dagger}-f_{1}^{\dagger}\right), \tilde{f}_{2}^{*}$ $=\left(f_{2}^{\dagger}\right) /\left(f_{1}^{\dagger}-f_{2}^{\dagger}\right)$. The two solutions give rise to different $\varepsilon$ 's,

$$
\begin{gathered}
\varepsilon=\left|\left(p_{n}+q_{n}\right) \tilde{f}_{1}-p_{n}\right|=\left|\left(p_{n}+q_{n}\right) \tilde{f}_{2}-q_{n}\right|, \\
\varepsilon^{*}=\left|\left(q_{n}-p_{n}\right) \tilde{f}_{1}^{*}-p_{n}\right|=\left|\left(q_{n}-p_{n}\right) \tilde{f}_{2}^{*}-q_{n}\right|,
\end{gathered}
$$

from which one can obtain $\varepsilon / \varepsilon^{*}=\left|\left(f_{2}-f_{1}\right) /\left(f_{1}+f_{2}\right)\right|<1$. So in this sense the $\left(\widetilde{f}_{1}, \widetilde{f}_{2}\right)$ solution is better than the $\left(\tilde{f}_{1}^{*}, \tilde{f}_{2}^{*}\right)$ solution. 


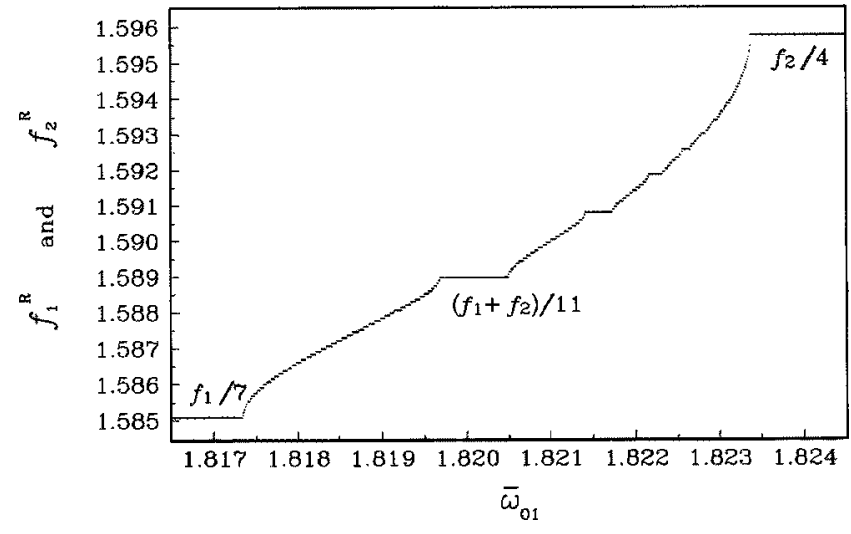

FIG. 3. The three-frequency devil's staircase for the system of ordinary differential equations of Eq. (15). The system parameters are equivalent to those of Fig. 2(b).

Now sticking with the $\left(\widetilde{f}_{1}, \widetilde{f}_{2}\right)$ solution, $p_{n},\left(p_{n}+q_{n}\right)$, and $q_{n}$ are obtained from the continued fraction expansions of $\tilde{f}_{1}$ and $\tilde{f}_{2}$. Since

$$
\begin{aligned}
& \widetilde{f}_{1}=\frac{f_{1}^{\dagger}}{f_{1}^{\dagger}+f_{2}^{\dagger}}=\frac{f_{1}}{f_{1}+f_{2}}=\frac{1}{1+\frac{1}{\frac{f_{1}}{f_{2}}},} \\
& \widetilde{f}_{2}=\frac{f_{2}^{\dagger}}{f_{1}^{\dagger}+f_{2}^{\dagger}}=\frac{f_{2}}{f_{1}+f_{2}}=\frac{1}{1+\frac{f_{1}}{f_{2}}},
\end{aligned}
$$

if we have the continued fraction expansion of $f_{1} / f_{2}$ $=\left(a_{1}, a_{2}, a_{3}, \ldots\right)$, that of $\widetilde{f}_{1}=\left(a_{1}+1, a_{2}, a_{3}, \ldots\right)$, and $\widetilde{f}_{2}$ $=\left(1, a_{1}, a_{2}, a_{3}, \ldots\right)$. Hence if $p_{n} / q_{n}$ is the $n$th strong convergent of $f_{1} / f_{2}$, or equivalently of $\widetilde{f}_{1} / \widetilde{f}_{2}$, given by this continued fraction expansion, $p_{n} /\left(p_{n}+q_{n}\right)$ and $q_{n} /\left(p_{n}\right.$ $+q_{n}$ ) are the strong convergents of $\widetilde{f}_{1}$ and $\widetilde{f}_{2}$, respectively. If $p_{n} / q_{n}$ is such a convergent of $f_{1} / f_{2}$, we may define as generalized adjacents any pair of $\left(f_{i} / r_{i}, f_{j} / r_{j}\right)$, with $f \in \mathbb{R}$ and $r \in \mathbb{Z}$, that satisfy

$$
\left|f_{i} r_{j}-f_{j} r_{i}\right|=\left|f_{1} q_{n}-f_{2} p_{n}\right| .
$$

From this definition, the subharmonics $f_{1} / p_{n}$ and $f_{2} / q_{n}$ are generalized adjacents, and the mediant between them is

$$
\widetilde{f}_{s}=\frac{f_{1}+f_{2}}{p_{n}+q_{n}}
$$

which by extension from the two-frequency case we hypothesize to be the largest plateau between $f_{1} / p_{n}$ and $f_{2} / q_{n}$. Starting with $\left(\tilde{f}_{1}^{*}, \tilde{f}_{2}^{*}\right)$ instead of $\left(\widetilde{f}_{1}, \widetilde{f}_{2}\right)$, we obtain the generalized mediant $\widetilde{f}_{s}^{*}=\left(f_{2}-f_{1}\right) /\left(q_{n}-p_{n}\right)$; both $\widetilde{f}_{s}$ and $\tilde{f}_{s}^{*}$ are shown in Fig. 1(a). The generalized mediant operation

$$
\frac{f_{1}}{r_{i}} \oplus \frac{f_{2}}{r_{j}}=\frac{f_{1}+f_{2}}{r_{i}+r_{j}}
$$

(a)

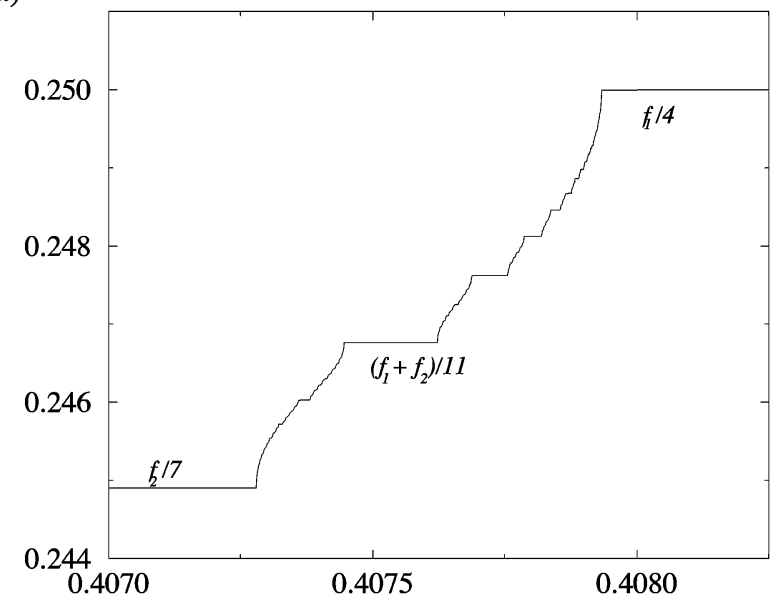

(b)

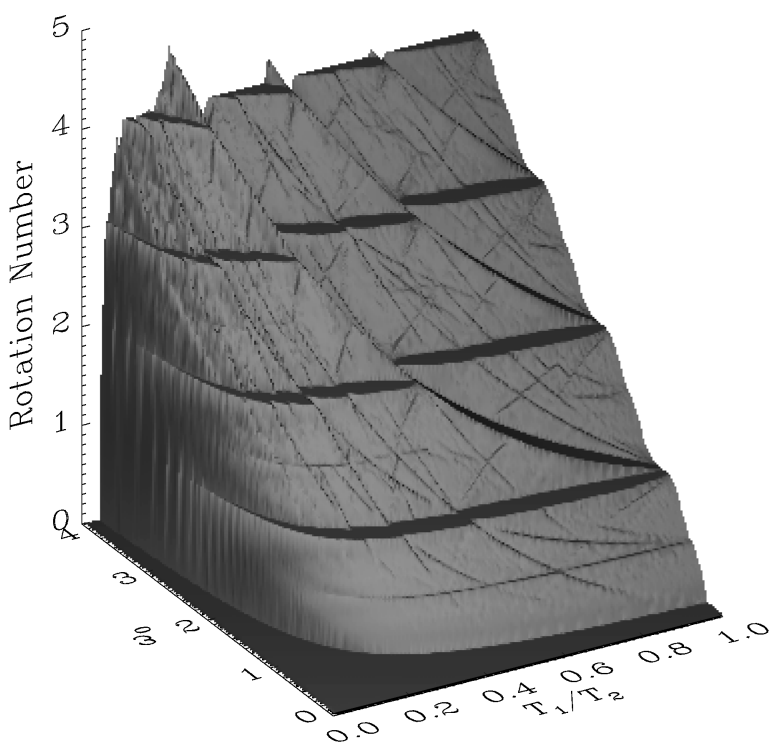

FIG. 4. Quasiperiodically forced circle map: (a) Threefrequency devil's staircase for forcing frequencies $f_{1}=1, f_{2}=12 / 7$. (b) Devil's ramps: the rotation number as a function of the external frequency ratio and the intrinsic frequency shows the global organization of three-frequency resonances.

then provides us with a generalized Farey tree for three frequencies; in Fig. 1(b) we show the first three levels of the tree obtained by recursive application of Eq. (14) to the adjacents $f_{1} / p_{n}$ and $\widetilde{f}_{s}^{*}$.

Let us take as an example a three-frequency system with the two external frequencies set to $f_{1}=2100 \mathrm{~Hz}$ and $f_{2}$ $=3600 \mathrm{~Hz}$. The frequency ratio $f_{1} / f_{2}$ is then $7 / 12$. The continued fraction expansion for $f_{1} / f_{2}$ is $(1,1,2,1,1)$, and the different truncations of this produce the convergents of $7 / 12$, which are 1/1, 1/2, 3/5, and 4/7. So we take $4 / 7$ as an approximation to the higher-order rational $7 / 12$, or equivalently in terms of the original frequencies, $f_{1} / f_{2}=2000$ $\mathrm{Hz} / 3500 \mathrm{~Hz}$ approximates $f_{1} / f_{2}=2100 \mathrm{~Hz} / 3600 \mathrm{~Hz}$ with a detuning of $100 \mathrm{~Hz}$. Between the adjacents $f_{1} / 4$ $=525 \mathrm{~Hz}$ and $f_{2} / 7=514.3 \mathrm{~Hz}$ lies the mediant $\widetilde{f}_{s}=\left(f_{1}\right.$ 
$\left.+f_{2}\right) / 11=518.2 \mathrm{~Hz}$, which we have hypothesized to be the widest resonance in this interval, and recursively applying the mediant operation gives us the Farey tree that predicts the entire hierachy of resonances in the interval. We now proceed to test this hypothesis in three-frequency dynamical systems.

We have constructed the three-frequency electronic oscillator shown in Fig. 2(a) [16]. Two phase-locked loop (PLL) circuits made up of voltage-controlled oscillators (VCO's) are coupled through a lowpass and integrator network and forced with two independent periodic forces. Typically, the outputs of the two oscillators are synchronized $1 / 1$. We filter the outputs of the two oscillators in order to attenuate the components at the external frequencies and we measure the output frequencies of both oscillators. If the two values coincide we plot them against the dc offset of oscillator 1, which is used as the control parameter (for the circuit a variation in the mean value of the $i$ th external force is equivalent to a linear change in the natural frequency of the $i$ th oscillator). The results are presented in Fig. 2(b) for the interval $\left(f_{2} / 7, f_{1} / 4\right)$ for $f_{1}$ and $f_{2}$ fixed at $2100 \mathrm{~Hz}$ and 3600 $\mathrm{Hz}$, respectively. This appears to be a typical devil's staircase familiar from periodically driven oscillators. It is, however, a three-frequency devil's staircase: the plateaus correspond to solutions with three linearly dependent basic frequencies (the frequency plotted plus the two forcing frequencies) instead of to periodic solutions. As predicted, the generalized mediant $\widetilde{f}_{s}=518.2 \mathrm{~Hz}$ is the largest resonance in the interval; moreover, the generalized Farey tree shown in Fig. 2(c) gives the entire hierarchy of resonances of Fig. 2(b).

We have integrated an exactly soluble system of ordinary differential equations with three interacting frequencies $[2,4]$

$$
\ddot{x}_{i}+\left(4 b x_{i}^{2}-2 a\right) \dot{x_{i}}+b^{2} x_{i}^{5}-2 a b x_{i}^{3}+\left(\omega_{0 i}(t)^{2}+a^{2}\right) x_{i}=f_{i}(t)
$$

for $i=1,2$. The external forces and the coupling term are chosen in such a way as to preserve the piecewise integrability of the overall system. The oscillators are coupled parametrically, their intrinsic frequency changing every time the coordinate of one of them changes sign $\omega_{0 i}(t)=\tilde{\omega}_{0 i}$ $+\operatorname{sgn} u_{i}(t) \operatorname{sgn} u_{j}(t) \Delta_{i}$. The $i$ th oscillator is driven by an impulsive external force $f_{i}(t)=V_{E i} \Sigma_{n} \delta\left(t-n \tau_{E i}\right)$ of frequency $\omega_{i}$ whose effect is to produce a discontinuity $V_{E i}$ in the oscillator velocity at times $n \tau_{E i}=n /\left(2 \pi \omega_{i}\right)$. We have performed a power spectrum analysis of the output of both oscillators while varying the intrinsic frequency of oscillator 1. We display in Fig. 3 the most prominent peak in each spectrum against $\tilde{\omega}_{01}$ for a parameter region equivalent to that of Fig. 2(b), and in which the two oscillators are also synchronized 1/1. All detectable resonances are again well described by the Farey tree structure of Fig. 2(c).

Our final example of a three-frequency system is the quasiperiodically forced circle map,

$$
\phi^{\prime}=\phi+\Omega_{n}+\frac{k}{2 \pi} \sin 2 \pi \phi \bmod 1
$$

The quasiperiodic sequence $\Omega_{n}$ is the time interval between successive pulses of a sequence composed of the superposition of two periodic subsequences, one of period equal to one and the other of period $T_{1} / T_{2}<1$ (with no loss of generality), multiplied by the value of the intrinsic frequency of the oscillator. In Fig. 4(a) we demonstrate that, for the same input frequencies as in the previous two cases, the output in the form of a devil's staircase is qualitatively unchanged; once again its organization is given by the generalized Farey tree. Moreover, Fig. 4(b) shows how three-frequency resonances are arranged globally in the form of devil's ramps in the parameter space.

By generalizing the known Farey tree structure of twofrequency resonances for three frequencies we have predicted the organization of three-frequency resonances in dynamical systems with three interacting frequencies. Our results for three different three-frequency dynamical systems for the same ratio of forcing frequencies $f_{1} / f_{2}$ show that in each example the predicted generalized Farey tree hierarchy is observed. We have repeated these experiments and simulations for different frequency ratios, both rational, as the example presented here, and irrational, for example, $f_{1} / f_{2}$ $=(1+\sqrt{5}) / 2$, the golden ratio. In every case we have examined, the local ordering of resonances around the convergents in the devil's staircase-slices through the devil's ramps of Fig. 4(b) - is well described by the generalized Farey tree hierarchy. We conclude that the organization we have described here is widespread, and conjecture that it may be universal in a large class of three-frequency systems.

JHEC and OP acknowledge the financial support of the Spanish Dirección General de Investigación Científica y Técnica, Contracts Nos. PB94-1167 and PB94-1172.
[1] C. Huygens, J. des Scavans, No. 11, Mar. 16; No. 12, Mar. 23 (1665)

[2] D.L. González and O. Piro, Phys. Rev. Lett. 50, 870 (1983).

[3] D.G. Aronson, M.A. Chory, G.R. Hall, and R.P. McGehee, Commun. Math. Phys. 83, 303 (1983).

[4] D.L. González and O. Piro, Phys. Rev. Lett. 55, 17 (1985).

[5] P. Cvitanović, B. Shraiman, and B. Söderberg, Phys. Scr. 32, 263 (1985).

[6] B.-L. Hao, Elementary Symbolic Dynamics and Chaos in Dis- sipative Systems (World Scientific, Singapore, 1989).

[7] D.K. Arrowsmith, J.H.E. Cartwright, A.N. Lansbury, and C.M. Place, Int. J. Bifurcation Chaos Appl. Sci. Eng. 3, 803 (1993).

[8] C. Baesens, J. Guckenheimer, S. Kim, and R.S. MacKay, Physica D 49, 387 (1991).

[9] R. van Buskirk and C. Jeffries, Phys. Rev. A 31, 3332 (1985).

[10] A.W. Cumming and P.S. Linsay, Phys. Rev. Lett. 60, 2719 (1988).

[11] P.S. Linsay and A.W. Cumming, Physica D 40, 196 (1989). 
[12] A. Y. Kinchin, Continued Fractions (University of Chicago Press, 1964).

[13] G. H. Hardy and E. M. Wright, An Introduction to the Theory of Numbers, 4th ed. (Oxford University Press, New York, 1975).
[14] S. Kim and S. Ostlund, Phys. Rev. Lett. 55, 1165 (1985).

[15] S. Kim and S. Ostlund, Phys. Rev. A 34, 3426 (1986).

[16] O. Calvo et al., IEEE Trans. Circuits Syst. (to be published). 